\title{
Is This Robot Like Me? Links Between Human and Robot Personality Traits $^{1}$
}

\author{
Sarah Woods, Kerstin Dautenhahn, Christina Kaouri, René te Boekhorst, Kheng Lee Koay \\ Adaptive Systems Research Group, School of Computer Science \\ University of Hertfordshire, United Kingdom \\ \{s.n.woods, k.dautenhahn\}@herts.ac.uk
}

\begin{abstract}
A relatively unexplored question for human-robot social interaction is whether a robot's personality should match that of the human user, or be different in the sense that humans do not want the robot to be like them. In this study, 28 adults interacted individually with a non-humanoid robot that demonstrated two robot behaviour styles (Socially Interactive, Socially Ignorant) in a simulated living room situation. Questionnaires assessed the extent to which adult ratings of their own personality traits were similar or different to the two robot behaviours. Results revealed that overall subjects did not view their own personality as similar to either of the two robot behaviour styles. Subjects viewed themselves as having stronger personality characteristics compared to the two robot behaviour styles. Important group differences were found, factors such as subject gender, age and technological experience were important in how subjects viewed their personality as being similar to the robot personality. Design implications for future studies are discussed.
\end{abstract}

Index Terms: Human-robot interaction, personality traits, social interaction, robot companion, Eysenck model of personality

\section{INTRODUCTION}

There are mixed opinions as to how humanlike a robot should be designed to engage in believable human-robot interactions $[1,2]$, and there are many design considerations to be taken into account both in terms of physical appearance and behavioural competencies [3]. The personality of a social robot is an important research domain for robot designers and has received only limited attention to date. There is no universally accepted definition of personality, but it can be broadly defined as a collection of individual differences, dispositions and temperaments that have consistency across situations and time [1,4]. Research studies have found that people tend to assign personality attributes to computers, agents, and robots which could assist the user in understanding its behaviour by shaping the users' expectations about the interaction experience $[3,5,6]$.

Those studies that have considered personality in relation to human-robot interaction have indicated that the personality of a robot should match its design purpose. For example, Goetz and Kiesler [7] found that people enjoyed robot interactions more with a happy robot, but were more likely to follow instructions from a serious robot. Also, correlations have been found between people's personalities and a virtual agent's social behaviour style [8]. In the same essence that people are uncomfortable with human personality styles that are unpredictable and inconsistent with situational contexts, it would follow that this relationship could be the same between human-robot interactions. To determine whether this relationship exists in human-robot interactions, it could be the case that humans try to match and project their own personality attributes and styles to that of a robot to create an engaging interaction that they feel at ease with and can make sense of. Alternatively, humans may not want to perceive themselves as being similar to a robot in terms of personality attributes. If this is the case they may infer different personality traits, or no personality traits, in the fear of losing their own identity, and wanting to remain unrelated to a robot.

The measurement of personality remains an area of controversy as there are many debates on the number of dimensions that define personality [9-12]. The widely used $\mathrm{PEN} / 3$ factor model of personality assessment was chosen for the present study [11]. Eysenck's view of personality was that people do not fit discrete categories, but instead there are dimensions of personality on which all individuals differ. Based on extensive research studies, the PEN model of personality is comprised of personality traits which intercorrelate and make up superfactors called "types", termed extraversion (E), neuroticism $(\mathrm{N})$ and psychoticism $(\mathrm{P})$. The extraversion vs. introversion factor is associated with the degree to which a person is outgoing and participative in relating to others. Neuroticism vs. emotional stability is related to an individual's adjustment to the environment and stability of behaviour over time. Finally, psychoticism is related to the loss of distortion of reality and the inability to distinguish reality and fantasy. Psychoticism is not a dimension like the other two factors, but is said to be present to some degree in all individuals. The three superfactors are independent dimensions. Our analysis in this paper is inspired by the PEN model to explore the relationship between users' personality traits, and their perceptions of robot personality traits, for two behaviour styles.

\section{Research Questions}

Research findings suggest that a robot's behaviour should fit the context and its task performance. Further, there is evidence that humans use personality as a social tool for

\footnotetext{
${ }^{1}$ The work described in this paper was conducted within the EU Integrated Project COGNIRON ("The Cognitive Robot Companion") and was funded by the European Commission Division FP6-IST Future and Emerging Technologies under Contract FP6-002020.
} 
interpreting and explaining others behaviour. Although research has shown that humans do attribute personality traits to robots, we are not aware of any research that has considered the nature of the assignment of these traits, and the possible relationship between the user and robot. For example, do humans assign their own personality traits to explain a robot's behaviour and intentions or assign contrasting personality traits in an effort to keep their own personality and identity separate to a non-living robot? This could have design implications in terms of matching robot behaviour and interaction style with desirable personality traits from a user perspective. The research questions for this study were:

1) Are there significant differences between participant personality traits and assigned robot personality traits?

2) Is there a relationship between human and robot personality?

3) If humans do project their own personality onto robots, does this attribution depend on the way the robot behaves?

4) What are the design implications for robots based on the findings from personality theory?

To investigate these research questions, an experiment was conducted where adults interacted with a robot in two different contexts (Negotiated Space Task and Assistance Task) for two contrasting robot behaviour styles (Socially Ignorant, Socially Interactive), in a simulated living room scenario. Participants completed questionnaires both pre and post robot interaction to assess their own personality, and the personality of the robot, based on Eysenck's PEN model.

\section{METHOD}

Design: Single human participants took part in this study in a simulated living room scenario at the University of Hertfordshire during July and August 2004 (see fig. 1). A commercially available, human-scaled PeopleBot ${ }^{\mathrm{TM}}$ robot was used to evaluate differences in participants' social behaviour and interaction styles when the robot displayed two contrasting behaviours (Socially Ignorant, Socially Interactive). The simulated living room was supervised by an experimenter at all times. The role of the experimenter was to explain the robot trials to the subject. If the subject initiated an interaction and wanted to ask a question, only then would the experimenter respond. Questionnaires that are relevant to this part of the study were an introductory questionnaire about subject demographics, subject personality questionnaire and the robot personality questionnaire.

\section{Sample characteristics: See Table 1}

Experimental Procedure: Introduction - A general welcome phase where the robot was introduced to the subject when they entered the simulated living room. An information sheet was given to the subject to read, along with a consent form to be signed, an Introductory Questionnaire and a Subject Personality Questionnaire to be completed. The robot moved around the room whilst the subject completed these initial questionnaires to familiarise them to the robot.

The Main Trial consisted of two tasks, a Negotiated Space Task and an Assistance Task (see fig. 2), which were repeated with the two contrasting robot behaviour styles (Socially Ignorant and Socially Interactive). The Negotiated Space Task involved the robot moving in the room (either with a Socially Ignorant or Socially Interactive behaviour style) while the subject went through a pile of books placed on a table, remembering one title at a time, walking over and writing down each title on the whiteboard. The Assistance Task involved the subject sitting at a table copying book titles from a whiteboard onto a piece of paper and underlining specific letters with a red/highlighter pen. The robot was responsible for bringing the missing red/highlighter pen to the table. The two tasks were chosen as they match the two key scenarios studied in the Cogniron project. At the end of these two tasks, the subject completed a robot personality questionnaire. The main trial was then repeated with the alternate robot behaviour style. Trials lasted for approximately one hour.

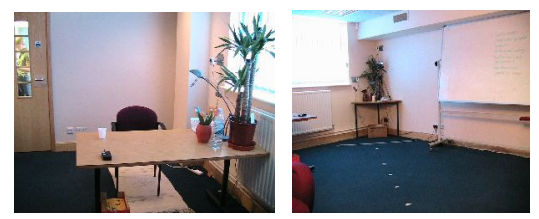

Fig. 1 Simulated living room for the human-robot interaction trials

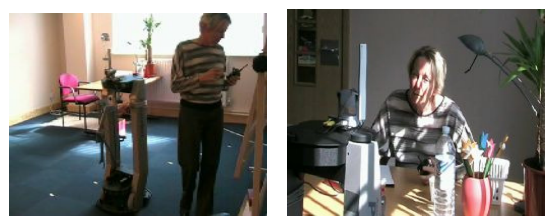

Fig 2. The negotiated space (left) and assistance task in the robot trials.

TABLE I: Sample Characteristics

\begin{tabular}{|l|c|}
\hline \multicolumn{2}{|c|}{ Sample Characteristics (N: 28): Recruited from University of Hertfordshire } \\
\hline Gender & $50 \%$ \\
Male & $50 \%$ \\
Female & $7 \%$ \\
\hline Age & $43 \%$ \\
$<25$ (but over 18 years) & $29 \%$ \\
$26-35$ & $11 \%$ \\
$36-45$ & \\
$46-55$ & $39 \%$ \\
\hline Occupation & $43 \%$ \\
Student & $18 \%$ \\
Academic/faculty staff & $50 \%$ \\
Researcher & $50 \%$ \\
\hline Educational/career background & \\
Technology related & \\
Non technology related (e.g. law) & \\
\hline
\end{tabular}

The Final Phase involved the subjects completing several questionnaires.

The questionnaires relevant to this study were:

a) Cogniron Introductory Questionnaire: This enquired about participants' personal details (age, gender, occupation), level of familiarity with robots, prior experience with robots (at work, as toys, in movies/books, in TV shows, in museums or in schools), and level of technical knowledge of robots were rated according to a 5-point Likert scale.

b) The Subject Personality Questionnaire - This was based on selected traits from Eysenck's three personality factors [13]: 1) neuroticism vs. emotional stability (anxiety, tension, shyness, emotional vulnerability), extraversion vs. introversion (sociability, general activity level, assertiveness, excitementseeking, dominance), and psychoticism (aggressiveness, impulsiveness, creativity). Some of Eysenck's traits were considered unsuitable for self-assessment (e.g. antisocial). 
Moreover, we considered only traits that could be rated for both human and robot personality. Autonomy was an additional trait added by our research team. Subjects were required to rate themselves for each of the 13 different personality traits using a 5-point Likert scale (e.g. for autonomy, continuous scale from $1=$ prefer being told what to do to $5=$ prefer to decide myself what to do).

c) The Robot Personality Questionnaire: This questionnaire followed a similar format to the subject personality questionnaire using the 5-point Likert scale, and included the following personality traits from Eysenck's model: anxiety, tension, shyness, emotional vulnerability, sociability, general activity level, assertiveness, excitement-seeking, dominance, aggressiveness, impulsiveness and creativity. A number of personality characteristics were added to the study and included: autonomy, intentionality, predictability of behaviour, controllability, and considerateness. Robot autonomy was measured from 'seemed to do what it was told/programmed to do' to 'seemed to make its own decisions'.

\section{Robot Behaviour Styles}

Subjects were exposed to two different robot behaviour styles and their reactions were recorded for the different situations. A counterbalanced design was used to avoid habituation effects, where the same task scenarios were used to test both robot behaviour conditions [Socially Ignorant (A), Socially Interactive (B)], which were defined a priori by the research team. The robot behaviour styles (A) and (B) constituted different behaviours for the Negotiated Space Task, and the Assistance Task (Tables II \& III)

TABLE II: Robot behaviour styles for negotiated space task

\begin{tabular}{|l|c|c|}
\hline Negotiated Space Task & Socially Ignorant (A) & Socially Interactive (B) \\
\hline Path & Straight line & $\begin{array}{c}\text { Circuitous route with respect to } \\
\text { subject's pose }\end{array}$ \\
\hline Speed & Fast & $\begin{array}{c}\text { Slow when close to subject, most } \\
\text { hesitant }\end{array}$ \\
\hline Camera & Static \& facing forward & $\begin{array}{c}\text { Moving \& tracking (showing } \\
\text { interest in subject's task) }\end{array}$ \\
\hline Encounter & $\begin{array}{c}\text { "excuse me", continue as } \\
\text { soon as possible }\end{array}$ & $\begin{array}{c}\text { "After you", continues after subject } \\
\text { has moved away }\end{array}$ \\
\hline
\end{tabular}

The robot behaviour styles were always referred to as behaviours $\mathrm{A}$ and $\mathrm{B}$ to ensure the experimenter did not give away procedural clues. The Socially Ignorant (A) behaviour style was expressed when the robot made little or no change to its behaviour when the participant was present. This corresponds to a robot treating a human not 'special' in any way but simply as an obstacle. In contrast, the Socially Interactive (B) behaviour style was classified if the robot took human presence into account by modifying 'robot optimum behaviour' (e.g. for a robot to go from $\mathrm{x}$ to point $\mathrm{y}$, the 'optimum' behaviour in an uncluttered environment is a straight line). A socially interactive robot was thus designed to be 'considerate' towards the subject. A mixture of autonomous programmes (e.g. wandering) and Wizard of $\mathrm{Oz}$ (WoZ) remote control was used.

TABLE III: Robot behaviour styles for Assistance Task

\begin{tabular}{|c|c|c|}
\hline Assistance Task & Socially Ignorant (A) & Socially Interactive (B) \\
\hline \multicolumn{3}{|c|}{$\mathbf{1}^{\text {st }}$ Encounter } \\
\hline Action & Does not wait for subject & $\begin{array}{c}\text { Waits for subject to look/ask } \\
\text { for pen }\end{array}$ \\
\hline Speech & $\begin{array}{c}\text { "I notice you need a pen. } \\
\text { I'll go fetch one" }\end{array}$ & $\begin{array}{c}\text { "I notice you need a pen. I'll } \\
\text { go fetch one" }\end{array}$ \\
\hline \multicolumn{3}{|c|}{$\mathbf{2}^{\text {nd }}$ Encounter } \\
\hline
\end{tabular}

\begin{tabular}{|c|c|c|} 
Action & $\begin{array}{c}\text { Bringing basket with pen to } \\
\text { side of table, close to } \\
\text { subject, putting it down }\end{array}$ & $\begin{array}{c}\text { Waiting in front of table facing } \\
\text { subject, waiting for subject to } \\
\text { notice, then putting basket } \\
\text { down }\end{array}$ \\
\hline Speech & $\begin{array}{c}\text { "Here you are, please take } \\
\text { the pen" }\end{array}$ & $\begin{array}{c}\text { "Here you are, please take the } \\
\text { pen" }\end{array}$ \\
\hline
\end{tabular}

\section{RESULTS}

Differences in personality traits between subjects and robots

Paired sample t-tests were computed to determine whether there were any significant differences between subjects' and robot A and B personality characteristics. Subjects rated themselves as being significantly more sociable, shyer, more anxious, tenser, more creative, higher in excitement-seeking, more dominant, more aggressive, and more autonomous compared to both robots $\mathrm{A}$ and $\mathrm{B}$. Subjects also rated themselves as being more assertive compared to robot $\mathrm{A}$, more vulnerable compared to robot $\mathrm{B}$, and more impulsive compared to robot $\mathrm{B}$. All t-test results were significant at $\mathrm{p}</=$ $.01^{2}$. Fig. 3 illustrates means and standard deviations of personality characteristics, for subjects and both robots A \& B.

These results seem to suggest that overall, the adult subjects in the current trials did not view their own personality as being similar to the personality characteristics of the robot for both robot behaviour styles (i.e. Socially Ignorant, Socially Interactive). It was interesting that in most cases they felt that they had more 'extreme' or stronger personality characteristics compared to the robot for both positive and negative traits. This could be an indicator that they did not view the robot as having particularly prominent or strong personality characteristics. Subjects also did not distinguish between their ratings of the personality between robot $\mathrm{A}$ and $\mathrm{B}$, although they displayed different behaviours during the trials towards the two different behaviour styles.

\section{Overall relationship between subject and robot personality}

Pearson correlation coefficients were calculated to examine the overall relationship between subject and robot personality traits overall. Only one significant positive linear relationship was revealed between subject dominance and Socially Ignorant robot (A) dominance $[\mathrm{r}(26)=0.44 ; \mathrm{p}=$ 0.02]. This indicates that the more dominant subjects rated themselves as being on the subject personality questionnaire, the more dominant they perceived robot A as being (or vice versa). No significant correlations were found between subject personality and the Socially Interactive robot (B).

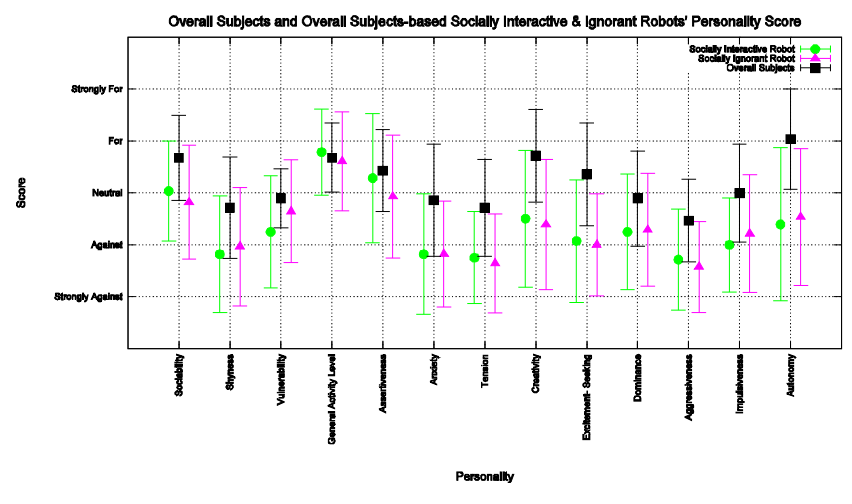

${ }^{2}$ Due to space limitations, individual t-test values are not shown 
Fig. 3 Mean and Standard deviations for personality characteristics for subjects and robots $\mathrm{A}$ and $\mathrm{B}$

The impact of gender in relation to subject and robot personality traits

Significant correlations were found between subject personality traits and ratings of robot personality for males and females. For males, the more anxious they rated themselves, the more anxious they rated robot $\mathrm{A}$, and the more psychotic males rated themselves, the more psychotic they rated robot $\mathrm{A}$. For females, a different pattern of findings emerged for links between their own personality and robot personality. The more assertive females rated themselves, the more assertive they rated robot $\mathrm{A}$, and the more dominant they rated themselves, the more dominant robot A was rated. No significant associations were revealed between subject personality and robot $\mathrm{B} .^{3}$

The impact of subject age in relation to subject and robot personality traits

For analysis purposes, two groups were formed, younger subjects were classified as $<35$ years, and older participants $>35$ years. Pearson correlation coefficients revealed only one significant negative linear relationship between older subjects and robot $\mathrm{A}$ for aggressiveness $[\mathrm{r}(14)=-0.62, \mathrm{p}=0.02]$. This finding indicates that the less aggressive older subjects rated their personality, the more aggressive they rated the personality of robot A.

For younger subjects, a different pattern of findings emerged. Five significant positive linear relationships were found between younger subjects' personality traits and robot A for assertiveness $[\mathrm{r}(14)=0.70, \mathrm{p}=0.005]$, anxiety $[\mathrm{r}(14)=$ $0.64, \mathrm{p}=0.02]$, aggressiveness $[\mathrm{r}(14)=0.55, \mathrm{p}=0.04]$, and impulsiveness $[\mathrm{r}(14)=0.57, \mathrm{p}=0.03]$. These findings show that the more assertive, anxious, aggressive and impulsive the younger subjects rated themselves, the more assertive, anxious, aggressive and impulsive they perceived the personality of Robot A. No significant relationships emerged for robot B for both older and younger subjects.

Technology related experience in relation to subject and robot personality traits

Subjects' level of technological experience was considered in relation to subject and robot personality traits. Firstly, no significant correlations were found between nontechnology background subjects, and their personality ratings and robot personality ratings. In contrast, a number of significant correlations were found between subjects who had a technology related background, their own personality ratings and robot personality ratings. The more anxious and aggressive subjects with a technological background rated themselves, the more aggressive and anxious they rated robot A. A negative linear relationship was found for general activity level, where the less active they perceived themselves, the more active they perceived robot A. Positive linear relationships were revealed for robot $\mathrm{B}$, i.e. the more anxious and excitement-seeking subjects rated themselves, the more excitement-seeking they perceived robot B. A negative linear relationship was found for shyness, where the less shy subjects

\footnotetext{
${ }^{3}$ Due to space limitations, not all statistical results can be shown
}

with a technology related background rated themselves, the shyer they rated robot B.

Overall, these findings seem to indicate differences between those subjects who had a technology-related background compared to those who did not. Subjects with a technology-related background appeared to relate to both robots $\mathrm{A}$ and $\mathrm{B}$ on a number of personality traits, suggesting perhaps that they believe that the robot has personality characteristics. However, the fact that no associations were found for the non-technology related subjects seems indicative that they did not view the robot as having any human-like personality characteristics.

The degree of personality attribution between subjects and the robot

To determine the degree to which subjects projected their own personality traits to the robot, the difference between the personality traits of each subject and how that same trait was evaluated by the subject to the robot (Socially Ignorant robot and Socially Interactive robot) was calculated. To allow for meaningful comparisons, differences were standardized to the score of the subject. In this way, we were able to obtain the differences for all traits $(j)$ and each of the $N$ subjects $(i)$. We then computed the mean discrepancy, standard error and the $95 \%$ confidence intervals $(C I)$ across each subject for the 13 personality traits discrepancies and for both robot behaviour styles. The mean discrepancy and the corresponding confidence intervals for the socially ignorant robot were plotted in fig. 4 against those of the socially interactive robot. The diagonal line indicates the positions where the degree of discrepancy between self-evaluation and attribution would be the same for both robot behaviour styles (i.e. $\Delta \bar{P}_{j}^{\text {sub,robotign) }}=$ $\left.\Delta \bar{P}_{j}^{\text {sub,robot(int) }}\right)$. Points that fall above the diagonal are characteristics that score relatively high for $\Delta \bar{P}_{j}^{\text {sub,robotign) }}$ and low for $\Delta \bar{P}_{j}^{\text {sub,robot(int) }}$.

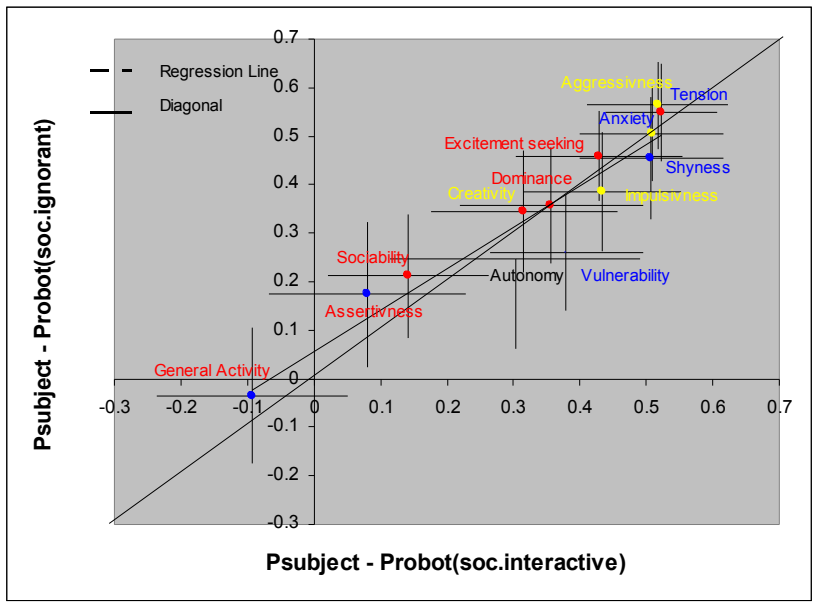

Fig 4. Discrepancies between subject values of personality traits and their attribution to robot behaviour styles A and B. Points are the average discrepancies calculated over measurements of 28 subjects \& $95 \%$ CI.

Fig. 4 illustrates some interesting features including: 1) The personality traits are relatively close to the diagonal. This indicates that the mean discrepancies measured for the Socially Ignorant robot are linearly related to those of the Socially Interactive robot. 2) The selected traits contributing to 
the 'neuroticism-emotional stability' and 'psychoticism' factor form a cluster and show larger discrepancies (for both robot behaviour styles) than those associated with the 'extravertintrovert' dimension. This suggests that subjects evaluated the robot as being more similar to themselves with respect to 'extra-introvert' traits than with 'neuroticism-emotional stability' and 'psychoticism' attributes, 3) Standard deviations are large; hence confidence intervals overlap for many trait discrepancies. This means that only a few of the trait discrepancies differ statistically among each other. Note, only 12 of the 27 traits used by Eysenck were measured in the present study. Further detailed statistical analysis (reported in [14]) suggested the 12 variables should be analysed in their own right, rather than focussing on the original Eysenck factors. Any reference to the 'Eysenck factors' in our results therefore strictly relates to the particular traits that we selected (possible alternative factors are proposed in [14]).

\section{DISCUSSION}

A summary of the main results of this study revealed that:

- For individual personality traits, subjects perceived themselves as having stronger personality characteristics compared to robots $\mathrm{A}$ and $\mathrm{B}$.

- Overall, subjects did not view their own personality as similar to robot behaviours A or B.

- Factors such as subject gender, age and technological experience were important in how subjects viewed their personality as being similar to the robot personality.

- The attribution of personality analysis revealed that subjects evaluated the robot as being more similar to themselves with respect to the traits contributing to the 'extra-introvert' factor compared to the 'neuroticism-emotional stability' and 'psychoticism' factors.

In response to research question one, a number of significant differences were found between subject personality ratings and the personality traits assigned to the robot behaviour styles. This implies that overall, subjects did not view their own personality as being similar to either the Socially Ignorant or Socially Interactive robot behaviour. In most cases, subjects felt that their personality was stronger for both positive and negative personality traits. This could mean that subjects did not view the robots as having a strong or identifiable personality. However, it should be noted that subjects participated in the trials only once within a simulated living-room situation, which does not fully resemble real-life scenarios. It would be interesting in future studies to consider the assignment of personality traits to the robots in more naturalistic surroundings, and longitudinally, to determine whether over time subjects build up a relationship with the robot and start to view it as having a more obvious personality.

Research question two considered the relationship between subject and robot personality. Although overall relationships between subjects' personality traits and the traits they assigned to the robot did not emerge, factors such as gender, age and technology-based experience were important. In the case of subject gender, relationships were different for males and females, although positive associations were found in all cases. Males and females appeared to interpret the robot behaviour and personality in different ways. This is an important future design consideration as it suggests that the desired personality and behaviour wanting to be conveyed by the robot may have very different meanings for males and females, and may lead to quite different human-robot interaction styles, and overall satisfaction with the experience.

A different pattern of findings also emerged according to subjects' age. For older subjects, only one negative relationship was found for aggressiveness ratings of the Socially Ignorant robot. However, for younger subjects, far more significant positive associations were found between subject personality and the personality of robot $\mathrm{B}$. The design implications of this finding suggest that the interaction experiences and interpretations between older and young subjects are very different. This of course could be related to previous exposure to robots, but could imply that older subjects were more anxious and wary of the robot interaction trials compared to younger subjects. If subjects are uncomfortable with interacting with a robot, this could result in them being unfavourable and less engaged towards robots, which might have negative marketing implications for the future of interactive robots, for example robot companions in the home. It is important that future studies examine the impact of age on robot interaction styles more closely and determine whether increased exposure to robots would help to reduce potential anxiety older subjects might have towards robots.

An important finding was that no significant associations were found for those subjects with limited /no technological background compared to a number of associations identified for those subjects from a technology related background. These findings indicate that subjects with no technological knowledge did not view either of the robot behaviour styles as having a personality. This could have important design implications as robot personality traits are likely to assist in human-robot interaction, as it could help the user e.g. to make sense of the interaction, leading to more engaging and believable interactions. Future studies on subjects with nontechnological backgrounds could explore the aspects of the robot they find the most and least accepting, and satisfying, and the reasons behind not thinking the robot had personality characteristics. This has implications if future robot companions capable of human-robot interaction are to be accepted by the wider community other than those people with a high interest in robots and technology. However, in the case of robots used e.g. as assistants or toys, it is very likely that people with a technological background will be a strong user group for any new robot product on the market.

The emerging pattern of findings for differences between age and technology related background could link to the argument posed in the introduction that some people may imbue their own personality onto the robot to help them understand, interpret and more fully engage in the interaction with the robot, whereas others may be fearful of losing their own identity and assuming that a robot can have similar personality characteristics and human qualities as them. Young people and those with a technological background seem to be more prepared to assign their own personality traits 
onto the robot compared to older subjects and those with a non-technology related background who wish to keep their own personality separate from that of a robot. These findings are related to those reported by Scopelliti et al. [15] that elderly subjects were more frightened at the prospect of having a robot in the home, and showed an element of distrust towards a robot in the home.

A further research question addressed was whether humans projected their own personality onto the robots and whether this depended on the way the robot behaved. Results showed that the degree of attribution of personal characteristics to the robot did not strongly depend on the robot behaviour style (i.e. Socially Interactive or Socially Ignorant) which indicates perhaps that subjects were unable to clearly distinguish the behaviours the robot was exhibiting and related personality characteristics. For example, in the current HRI trials, it could be case that the researchers felt it was polite (i.e. socially interactive) for the robot to wait until the subject looked at the robot in the Assistance Task for the pen, but some subjects commented that they found this irritating, and would therefore have maybe defined the behaviour as being socially ignorant. Future studies should consider in more detail the ability of subjects to distinguish between different types of robot behaviour and related personality constructs as it could help to shape human-robot interaction into a more enjoyable and satisfying experience. This could be achieved e.g. by carrying out detailed pilot studies where subjects use 'Think Aloud' strategies during robot interactions.

To conclude, results from our robot trials indicate that human subjects do not tend to assign their personality traits to match the robots'. This remained the case for different robot behaviour styles. However, subject gender, age and technological background were all important factors related to the extent to which subjects ascribed their own personality traits to the robot. It seems that younger subjects with technology related backgrounds were happy to ascribe their own personality traits to the robot, perhaps in an attempt to understand the interaction more fully. In contrast, older subjects with little technological background did not view their own personality as being similar to that of the robot, perhaps in an attempt to keep their own identity separate to that of the robot. This is a relatively unexplored area of human-robot interaction studies and future research needs to consider the role of robot personality in more detail to fully understand the contribution of personality in emulating engaging human-robot interactions.

\section{ACKNOWLEDGMENTS}

We would like to thank Mick Walters, Chrystopher Nehaniv, Iain Werry, David Lee, and all the participants for their involvement in this study.

\section{REFERENCES}

[1] C. L. Breazeal, Designing sociable robots. Massachusetts: The MIT Press, 2002.

[2] T. Minato, M. Shimada, H. Ishiguro, \& S. Itakura, "Development of an android robot for studying human-robot interaction," Lecture Notes in Computer Science, vol. 3029, pp. 424-434, 2004.

[3] S. Woods, K. Dautenhahn, \& J. Schulz, "The design space of robots: Investigating children's views," Proc. Ro-Man, Japan, 2004, pp. 47-52.
[4] D. C. Dryer, "Getting personal with computers: How to design personalities for agents," Applied Artificial Intelligence, vol. 13, pp. 273-295, 1999.

[5] D. A. Norman, "How might people interact with agents," Communications of the ACM, vol. 37, pp. 68-71, 1994.

[6] S. Woods, K. Dautenhahn, \& J. Schulz, "Child and adults' perspective on robot appearance," Proc. AISB 05, UK, 2005, pp. 126-132.

[7] J. Goetz \& S. Kiesler, "Cooperation with a robotic assistant," presented at CHI'02, New York, USA, 2002.

[8] J. Cassell \& J. Bickmore, "Negotiated collusion: Modeling social language and its relationship effects in intelligent agents," User Modeling and User-Adapted Interaction, vol. 13, pp. 89-132, 2003.

[9] P. T. Costa \& R. R. McCrae, "Personality stability \& its implications for clinical psychology," Clinical Psych. Review, vol. 6, pp. 407-423, 1986.

[10] H. J. Eysenck \& S. B. G. Eysenck, Manual of the Eysenck personality questionnaire. London: Hodder and Stoughton, 1975.

[11] H. J. Eysenck, "Dimensions of personality: 16, 5 or 3? Criteria for a taxonomic paradigm," Personality \& Ind Differences, vol. 12, pp. 773790, 1991.

[12] P. T. Costa \& R. R. McCrae, "Four ways five factors are basic," Personality \& Ind. Differences, vol. 13, pp. 653-665, 1992.

[13] H. J. Eysenck \& M. W. Eysenck, Personality \& ind. differences: A natural science approach. New York, USA: Plenum, 1985.

[14] M. L. Walters, K. Dautenhahn, R. te Boekhorst, K. L. Koay, C. Kaouri, S. Woods, C. L. Nehaniv, D. Lee, I. Werry, "The Influence of Subjects' Personality Traits on Personal Spatial Zones in a Human-Robot Interaction Experiment", Proc. IEEE Ro-man, 2005, pp. 347-352.

[15] M. Scopelliti, et al., "If I had a robot at home. Peoples' representation of domestic robots," in Designing a more inclusive world, S. Keates, et al. Eds. Cambridge, UK: Springer, 2004, pp. 257-266. 